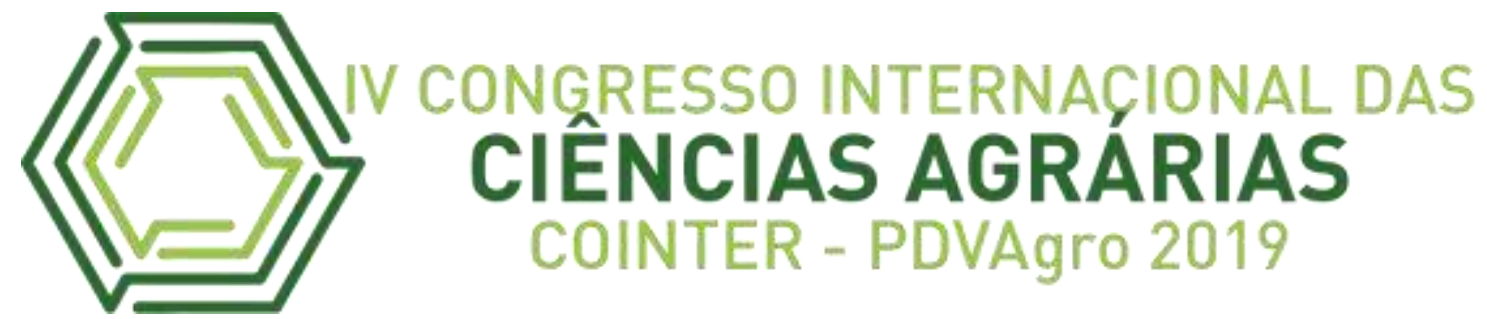

\title{
ÓLEOS ESSENCIAIS COM POTENCIAL ANTIOXIDANTE
}

ESENCIALES CON POTENCIAL ANTIOXIDANTE

ESSENTIAL OILS WITH ANTIOXIDANT POTENTIAL

\author{
Apresentação: Comunicação Oral \\ Antonia Flávia Costa Souto ${ }^{1}$; Lizienny Pinheiro Peixoto ${ }^{2}$; Daniel Yuri Xavier de Sousa $^{3}$ \\ Isis Alves Palmeira ${ }^{4}$; Allana Kellen Lima Santos Pereira ${ }^{5}$. \\ DOI:https://doi.org/10.31692/2526-7701.IVCOINTERPDVAgro.2019.0146
}

\begin{abstract}
Resumo
Os óleos essenciais são extraídos de plantas aromáticas, cujos constituintes químicos são predominantemente mono- e sesquiterpenos. Esses óleos essenciais são utilizados como flavorizantes aromatizantes e terapêuticos nas indústrias alimentícias, farmacêuticas e cosméticas. Geralmente, as plantas aromáticas são medicinais e estas se fazem presentes no cotidiano das pessoas com fins terapêuticos, sendo utilizadas na medicina popular por milênios. O objetivo dessa pesquisa é estudar o potencial antioxidante de óleos essenciais de espécies vegetais cultivadas no Ceará. A metodologia utilizada no teste de atividade antioxidante é a do sequestro de radicais livres, semelhante à descrita por Hegazi e Hady, onde o radical utilizado foi o DPPH (difenilpicril-hidrazil) na concentração de $60 \mathrm{mmol}$. Alguns óleos essenciais de plantas apresentaram bons resultados como antioxidantes. Ao serem comparados à vitamina $\mathrm{C}$, padrão, pode-se concluir que essas plantas são potenciais antioxidantes.
\end{abstract}

Palavras-chave: Química, óleo essencial, plantas e atividade antioxidante.

\section{Resumen}

Los aceites esenciales se extraen de plantas aromáticas, cuyos componentes químicos son predominantemente mono y sesquiterpenos. Estos aceites esenciales se utilizan como aromatizantes y aromatizantes terapéuticos en las industrias alimentaria, farmacéutica y

\footnotetext{
${ }^{1}$ Agronomia, Universidade Federal do Cariri, flaviasouto15@gmail.com

2 Engenharia de Materiais, Universidade Federal do Cariri, lizienny.peixoto@aluno.ufca.edu.br

${ }^{3}$ Agronomia, Universidade Federal do Cariri, yuri.xavier@aluno.ufca.edu.br

${ }^{4}$ Agronomia, Universidade Federal do Cariri, isis.palmeira@aluno.ufca.edu.br

${ }^{5}$ Doutora, Universidade Federal do Cariri, allana.lima@ufca.edu.br
} 




cosmética. En general, las plantas aromáticas son medicinales y están presentes en la vida cotidiana de las personas con fines terapéuticos, y se utilizan en la medicina popular durante milenios. El objetivo de esta investigación es estudiar el potencial antioxidante de los aceites esenciales de especies de plantas cultivadas en Ceará. La metodología utilizada en la prueba de actividad antioxidante es el secuestro de radicales libres, similar al descrito por Hegazi y Hady, donde el radical utilizado fue DPPH (difenilpicrylhidrazina) a una concentración de $60 \mathrm{mmol}$. Algunos aceites esenciales de plantas han mostrado buenos resultados como antioxidantes. En comparación con la vitamina estándar, se puede concluir que estas plantas son antioxidantes potenciales.

Palabras clave: Química, aceites esenciales, plantas y actividad antioxidante.

\begin{abstract}
Essential oils are extracted from aromatic plants, whose chemical constituents are predominantly mono- and sesquiterpenes. These essential oils are used as flavoring and therapeutic flavorings in the food, pharmaceutical and cosmetic industries. Generally, aromatic plants are medicinal and these are present in the daily lives of people for therapeutic purposes, being used in folk medicine for millennia. The objective of this research is to study the antioxidant potential of essential oils of plant species grown in Ceará. The methodology used in the antioxidant activity test is free radical sequestration, similar to that described by Hegazi and Hady, where the radical used was DPPH (diphenylpicryl-hydrazine) at a concentration of $60 \mathrm{mmol}$. Some plant essential oils have shown good results as antioxidants. When compared to standard vitamin $\mathrm{C}$, it can be concluded that these plants are potential antioxidants.
\end{abstract}

Keywords: Chemistry, essential oil, plants and antioxidant activity.

\title{
Introdução
}

Óleos essenciais (OE) são compostos complexos, caracterizados por forte odor, produzidos como metabólicos secundários de algumas, principalmente de mono e sesquiterpenos e de fenilpropanóides, metabólitos especiais que conferem suas características organolépticas (BIZZO, 2009). 


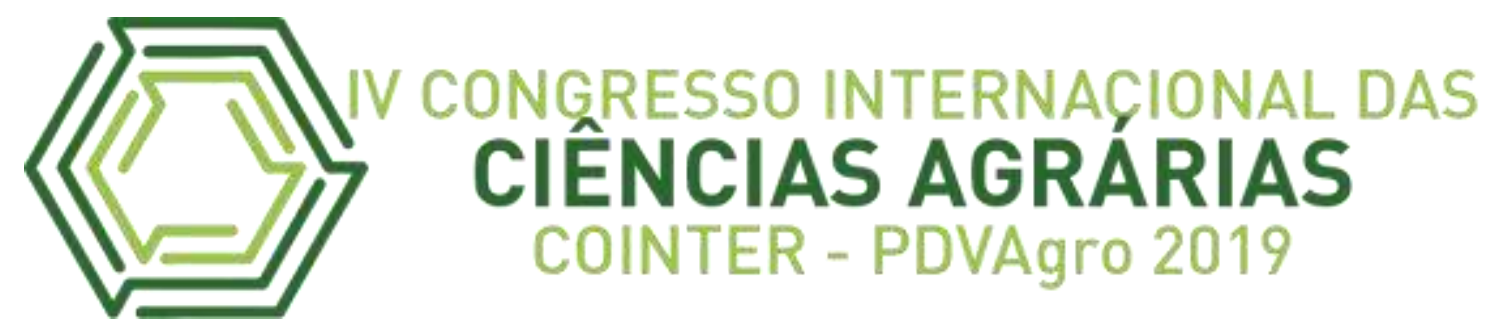

Os óleos essenciais são metabólitos secundários, por sua vez, podem ser extraídos de várias partes das plantas, sua composição química é complexa e garante aos vegetais vantagens de adaptação no meio em que estão introduzidos.

A produção de OE também está relacionada com várias funções necessárias para a sobrevivência das plantas, exercendo papel fundamental de defesa como antibacterianas, antivirais, antifúngicas e inseticidas. Tanto as folhas, inflorescências e cascas são exemplos de matérias-primas utilizadas para a extração dos óleos essenciais, estes, são de suma importância para a cosmética e perfumaria pelos seus aromas e fragrâncias, e medicamentos, na elaboração de produtos farmacêuticos. Uma das técnicas mais utilizada para a obtenção de óleo essencial é por arraste a vapor (BIZZO, 2009).

A constituição química dos óleos voláteis varia entre espécies e entre as partes de uma mesma planta. Uma mesma espécie botânica pode ser influenciada pelo local de cultivo, pelas condições de coleta, armazenamento, além dos fatores edafoclimáticos. Os componentes dos óleos essenciais são derivados principalmente de terpênicos, como os mono- e sesquiterpenos e os fenilpropanóides. Com base na possibilidade de se fazer uso dos óleos essenciais para aumentar a conservação e prolongar a validade de alimentos e a possível diminuição do uso de antioxidantes sintéticos a agentes antimicrobianos, muitos estudos têm se dedicado na demonstração de potenciais promissores de tais compostos no combate aos radicais livres e bactérias patogênicas, tornando o emprego desses metabólitos uma alternativa possível de forma ecológica e economicamente (MIRANDA,2016).

A comunidade científica tem empregado esforços com o intuito de esclarecer as diversas propriedades dos óleos essenciais. Atividades farmacológicas a exemplo da antinociceptiva, anticonvulsivantes e hipotensivas vêm sendo exposta na literatura para diversos óleos essenciais, como o estudo desenvolvido com OE de Cymbopogom winterianus e seus constituintes (BRITO et al.2013).

O objetivo desse trabalho foi determinar o potencial antioxidante de óleos essenciais de espécies vegetais aromáticas, tanto das suas folhas, quanto inflorescências: Gonçalo Alves (Astronium fraxinifolium), Cidreira (Melissa officinalis), Manjericão (Ocimum basilicum L.) e Capim-limão (Cymbopogon citratus). 


\section{CIEN CONGRESSO INTERNACIONAL DAS
CONTER - PDARARO 2019}

\section{Fundamentação teórica}

A fitoterapia, designada como o uso de plantas medicinais usadas no tratamento de doenças, faz parte da prática da medicina popular, constituindo um conjunto de saberes internalizados e transmitidos de geração a geração, como uma tradição. Contudo, tal prática diminuiu em decorrência ao processo de industrialização ocorrido no país entre as décadas de 1940 e 1950. Trata-se de uma forma eficaz de atendimento primário a saúde, podendo ser complementar ao tratamento usualmente empregado para a população de menor renda (BRUNING et al, 2012).

O estudo de plantas medicinais, a partir de seu emprego pelas comunidades em si, pode fornecer vastas informações úteis para a elaboração de estudos farmacológicos, fitoquímicos e agronômicos sobre estas plantas, com grande economia de tempo e de dinheiro. Desta forma, podemos planejar a pesquisa a partir do conhecimento empírico já existente, e por muitas vezes consagrado pelo uso contínuo, que deverá ser testado em bases científicas (BRASILEIRO, 2008).

As atividades essenciais das células dependem de energia que é obtida através de um processo metabólico, a oxidação. No entanto, quando o oxigênio é metabolizado pelas células vivas, desencadeia também a produção de radicais, que está diretamente associado ao processo de envelhecimento, e a doenças como Alzhimer, câncer e outras. A produção desses radicais é controlada por compostos antioxidantes nos seres vivos, este controle pode ser de forma endógena ou exógena (através, por exemplo, do alimento e de outras fontes como a vitamina $\mathrm{C}$ e E, polifenóis, carotenoides e selênio); os compostos tanto podem desativar os radicais, como estabiliza-los, antes que afetem as células alvo. Quando esses antioxidantes são limitados, pode vir a ocorrer lesões oxidativas, ademais, podem ser intensificadas ao decorrer do tempo, sendo assim, essas substâncias antioxidantes são de suma importância na diminuição dos efeitos maléficos dos radicais livres gerados em processos oxidativos no metabolismo humano (ROESLER et al, 2007).

Uma das maneiras de fazer a identificação do potencial antioxidante de um elemento é pelo método DPPH (2,2-difenil- 1-picril- hidrazil), neste modo, quando o material analisado possui a capacidade antioxidante, ele reage com o DPPH, formando o 


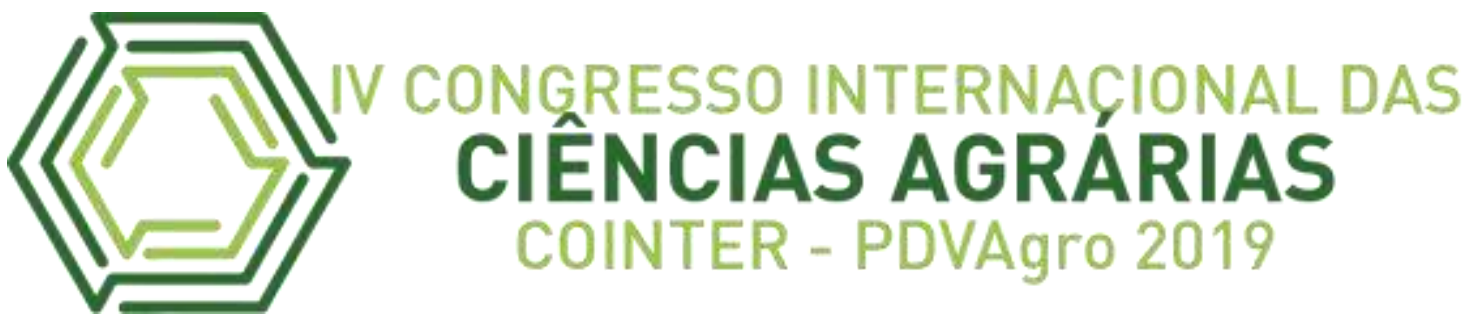

DPPH-H, sua forma reduzida, essa redução provoca a alteração de cor violeta para amarela (RODRIGUES, 2017).

É importante salientar que tem-se grandes perspectivas para o uso dos óleos essenciais, visto que sua utilização pode ser feita tanto pelas indústrias farmacêutica, como na indústria cosmética, que os aplica na produção de protetores solares, loções antissépticas, desodorantes, produtos de higiene e perfumes e também na indústria alimentícia, sendo usadas como aromatizantes e/ou conservantes, ou ainda na fabricação de alimentos e bebidas (BRITO, A. M. G). Onde muitas dessas propriedades deve-se ao caráter antioxidante dos componentes químicos dos óleos essenciais.

\section{Metodologia}

\section{Escolha da espécie estudada}

Realizamos inicialmente um levantamento bibliográfico de plantas aromáticas e dentre essas, selecionamos as que são cultivadas na região do Cariri. Em seguida selecionamos preferencialmente as que são consideradas medicinais. Realizamos levantamento bibliográfico no Chemical Abstracts com o objetivo de obtermos informações a respeito de possíveis atividades biológicas e/ou farmacológicas das espécies escolhidas para o estudo, coleta e identificação do material botânico, este, foi coletado e constituído principalmente de folhas frescas das espécies escolhidas: Gonçalo Alves (Astronium fraxinifolium), cidreira (Melissa officinalis), manjericão (Ocimum basilicum L.) e o capim-limão (Cymbopogon citratus), tanto das suas folhas, quanto inflorescências . No ato da coleta é feita a exicata da amostra vegetal que consiste em separarmos as partes aéreas da planta com flores e frutos. Condição esta fundamental para a determinação inequívoca do gênero e espécie da planta, esta amostra é então enviada ao herbário da Universidade Regional do Cariri ou ao herbário da Universidade Federal do Ceará para a identificação do material vegetal. 


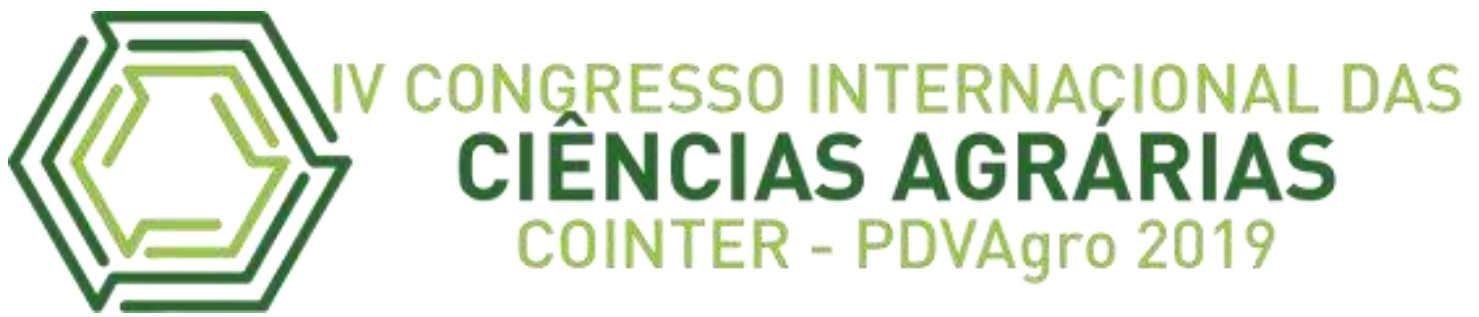

Para a obtenção dos óleos essenciais, o material botânico utilizado foi a parte aérea (folhas e inflorescências frescas), as quais foram submetidas à hidrodestilação em aparelho graduado Clevenger, usando-se $100 \mathrm{~g}$ de folhas, em $1000 \mathrm{~mL}$ de água destilada, em temperatura máxima de $100{ }^{\circ} \mathrm{C}$ até atingir a fervura, reduzimos posteriormente para $75{ }^{\circ} \mathrm{C}$, por um período de aproximadamente $2 \mathrm{~h}$ sob refluxo, variando de acordo com a espécie em questão. Ao final, os óleos essenciais foram coletados com uma pipeta, secos com sulfato de sódio anidro, armazenados em eppendorf protegidos da luz com papel alumínio e enviados para análise.

Atividade Antioxidante: método do sequestro do radical DPPH

Os óleos essenciais testados foram de Gonçalo Alves (Astronium fraxinifolium), Cidreira (Melissa officinalis), Manjericão (Ocimum basilicum L.) e Capim-limão (Cymbopogon citratus). A metodologia utilizada no teste de atividade antioxidante é a do sequestro de radicais livres, semelhante à descrita por Hegazi e Hady, onde o radical utilizado foi o DPPH (difenilpicril-hidrazil) na concentração de $60 \mathrm{mmol}$. Numa cubeta de $3 \mathrm{~mL}$ colocou-se $1000 \mu \mathrm{L}$ de DPPH, em seguida adicionou-se $1000 \mu \mathrm{L}$ de etanol (EtOH), então fez-se a leitura no ultravioleta (UV) cuja absorbância está em 520 nm, onde obteve-se uma absorbância "X".

Foram preparadas quatro amostras cujas concentrações foram 1,0 mg/mL, 0,5 $\mathrm{mg} / \mathrm{mL}, 0,25 \mathrm{mg} / \mathrm{mL}$ e $0,125 \mathrm{mg} / \mathrm{mL}$, onde foram colocadas $1000 \mu \mathrm{L}$ da amostra e adicionada $1000 \mu \mathrm{L}$ de DPPH na cubeta e levadas para o UV, para que fosse feita uma absorbância "Y". O declínio da concentração é registrado como declínio na absorbância em $\lambda_{\text {máx }} 520 \mathrm{~nm}$ por um período de 30 minutos. A absorbância correspondente a $100 \%$ de sequestro de radical é determinada com o teste realizado usando-se os controladores positivos Trolox (ácido 6-hidróxi-2,5,7,8-tetrametilcroman-2-carboxílico) e BHT (2,6-diterc-butil-4-metilfenol) (Figura 01), que causam o sequestro completo dos radicais livres. Neste teste foi utilizado o espectro eletrônico Hewlett Packard 8453 Diode-Array acoplado a um computador; para análise utilizou-se uma célula de quartzo de caminho ótico de 1,0 cm (HEGAZI, 2002). 


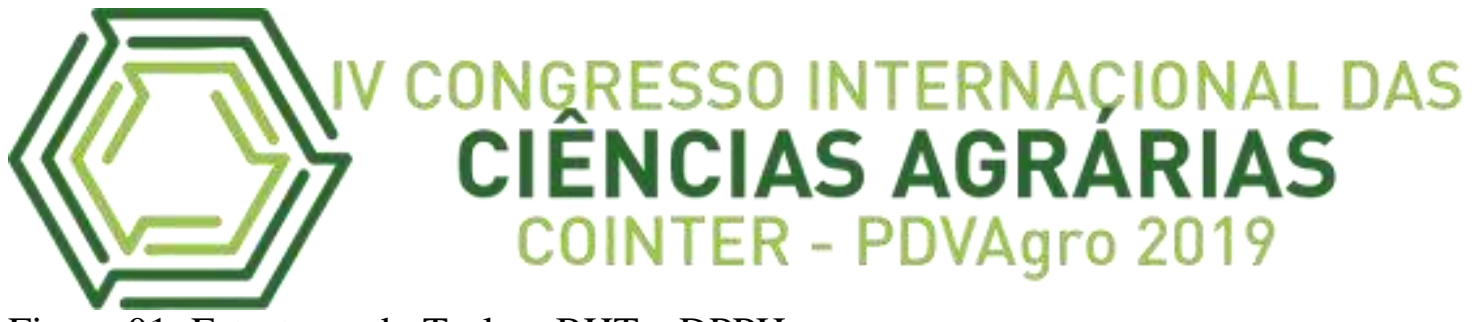

Figura 01: Estruturas do Trolox, BHT e DPPH<smiles>Cc1c(C)c2c(c(C)c1O)CCC(C)(C(=O)O)O2</smiles>

Trolox<smiles>Cc1cc(C(C)(C)C)c(O)c(C(C)(C)C)c1</smiles>

BHT

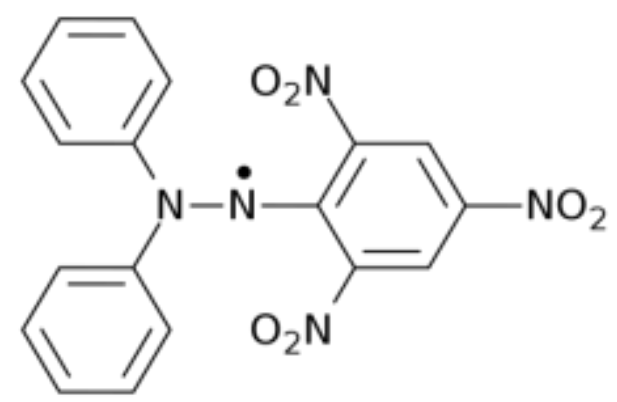

$\mathrm{DPPH}$

\section{Resultados}

Atividade Antioxidante: Gonçalo Alves

Para este teste, o método utilizado foi o método do sequestro de radicais livres que foi realizado em quatro concentrações (Tabela 01). O óleo apresentou um resultado ruim frente ao sequestro dos radicais livres com um percentual de 3,6 \% a uma contração de $0,5 \mathrm{mg} / \mathrm{ml}$, quando comparado ao padrão, vitamina $\mathrm{C}$ (Tabela 02), que apresentou um percentual de $88,55 \%$ para a concentração de $0,5 \mathrm{mg} / \mathrm{ml}$. Logo o OE de Gonçalo Alves não apresenta atividade antioxidante. 


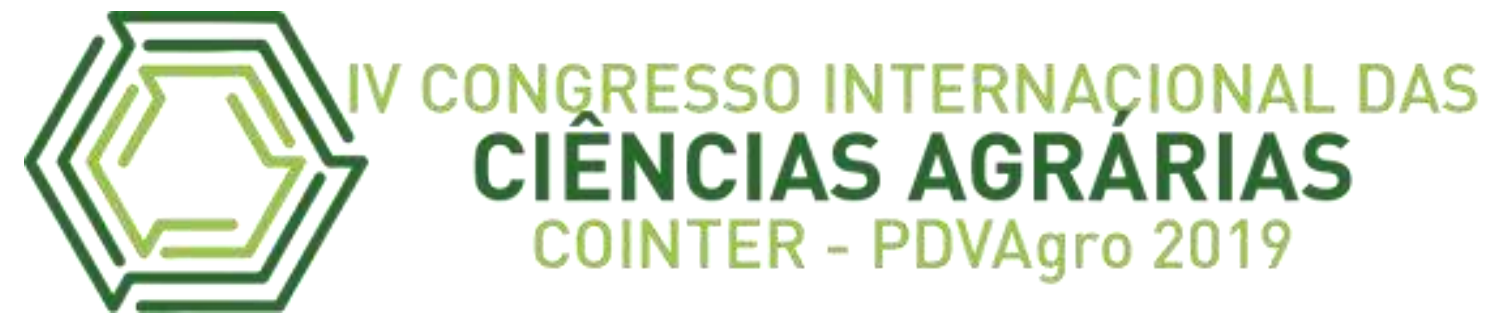

Tabela 01: Resultado do teste de atividade antioxidante do óleo essencial de Gonçalo Alves (Astronium fraxinifolium) com seu percentual de sequestro de radicais livres (inibição).

\begin{tabular}{|l|l|l|l|c|c|c|c|}
\hline Concentração(mg/ml) & Com. & \multirow{2}{*}{$\%$ Inib. } & \multicolumn{3}{|c|}{ Abs. } & Abs. & \\
\cline { 4 - 8 } & $(\mathrm{ppm})$ & & $\mathrm{x}$ & $\mathrm{y}$ & $\mathrm{z}$ & $\mathrm{x}, \mathrm{y}, \mathrm{z}$ & $\mathrm{r}$ \\
\hline 1 & 1000 & 3,398 & 0,199 & 0,199 & 0,199 & 0,199 & $3,4 \mathrm{E}-17$ \\
\hline 0,5 & 500 & 3,560 & 0,199 & 0,199 & 0,198 & 0,199 & 0,000577 \\
\hline 0,25 & 250 & 2,104 & 0,202 & 0,202 & 0,201 & 0,202 & 0,000577 \\
\hline 0,125 & 125 & 0,162 & 0,206 & 0,205 & 0,206 & 0,206 & 0,000577 \\
\hline & & & & & & & \\
\hline & & & & & & & \\
\hline DPPH & & & 0,206 & & & & \\
\hline
\end{tabular}

Fonte: Própria (2019)

Tabela 02: Resultado do teste de atividade antioxidante do padrão vitamina C com seu percentual de sequestro de radicais livres (inibição)

\begin{tabular}{|l|l|l|l|c|c|c|c|}
\hline Concentração(mg/ml) & Com. & \multirow{2}{*}{ \%Inib. } & \multicolumn{3}{|c|}{ Abs. } & Abs. & \\
\cline { 4 - 8 } & $(\mathrm{ppm})$ & & $\mathrm{x}$ & $\mathrm{y}$ & $\mathrm{z}$ & $\mathrm{x}, \mathrm{y}, \mathrm{z}$ & $\mathrm{r}$ \\
\hline 0,5 & 1000 & 88,557 & 0,023 & 0,022 & 0,024 & 0,023 & 0,001 \\
\hline 0,25 & 250 & 77,114 & 0,045 & 0,046 & 0,047 & 0,046 & 0,001 \\
\hline 0,125 & 125 & 61,194 & 0,079 & 0,078 & 0,077 & 0,078 & 0,001 \\
\hline 0,0625 & 62,5 & 50,746 & 0,100 & 0,099 & 0,098 & 0,099 & 0,001 \\
\hline 0,03125 & 31,25 & 37,148 & 0,125 & 0,127 & 0,127 & 0,126 & 0,001155 \\
\hline & & & & & & & \\
\hline DPPH & & & 0,201 & & & & \\
\hline
\end{tabular}

Fonte: Própria (2019)

\section{$\underline{\text { Atividade Antioxidante: Cidreira }}$}

Para este teste, o método utilizado foi o método do sequestro de radicais livres que foi realizado em quatro concentrações (Tabela 03). O óleo apresentou um baixo resultado frente ao sequestro dos radicais livres com um percentual de 33,5 \% para concentração de $1 \mathrm{mg} / \mathrm{mL}$ quando comparado ao padrão Vitamina C com um percentual de 88,5\% para 


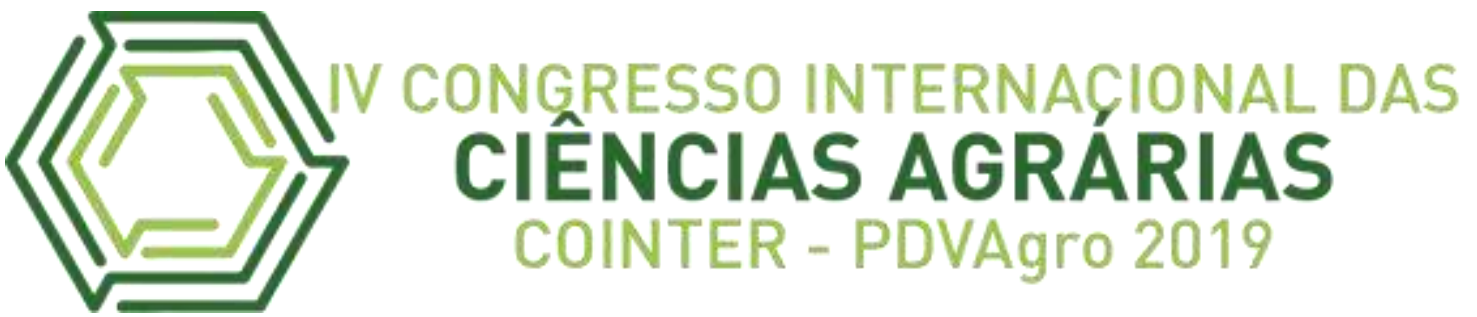

a concentração de $0,5 \mathrm{mg} / \mathrm{mL}$ (Tabela 02 ). $\mathrm{O}$ OE da cidreira não apresenta-se como um bom antioxidante.

Tabela 03: Resultado do teste de atividade antioxidante do óleo essencial de Cidreira (Melissa officinalis) com seu percentual de sequestro de radicais livres (inibição)

\begin{tabular}{|l|l|l|l|c|c|c|c|}
\hline Concentração(mg/ml) & Com. & \multirow{2}{*}{ \%Inib. } & \multicolumn{3}{|c|}{ Abs. } & Abs. & \\
\cline { 4 - 8 } & $(\mathrm{ppm})$ & & $\mathrm{x}$ & $\mathrm{y}$ & $\mathrm{z}$ & $\mathrm{x}, \mathrm{y}, \mathrm{z}$ & $\mathrm{r}$ \\
\hline 1 & 1000 & 33,499 & 0,133 & 0,133 & 0,135 & 0,134 & 0,001155 \\
\hline 0,5 & 500 & 18,574 & 0,166 & 0,164 & 0,161 & 0,164 & 0,002517 \\
\hline 0,25 & 250 & 10,945 & 0,178 & 0,179 & 0,180 & 0,179 & 0,001 \\
\hline 0,125 & 125 & 0,332 & 0,201 & 0,200 & 0,200 & 0,200 & 0,000577 \\
\hline & & & & & & & \\
\hline & & & & & & & \\
\hline DPPH & & & 0,201 & & & & \\
\hline
\end{tabular}

Fonte: Própria (2019)

Atividade Antioxidante: Manjericão

Para este teste, o método utilizado foi o método do sequestro de radicais livres que foi realizado em quatro concentrações $\mathrm{O}$ óleo apresentou um resultado excelente frente ao sequestro dos radicais livres com um percentual de $87,7 \%$ para a concentração de 1 mg/mL (Tabela 04). Quando comparamos o padrão, vitamina C (Tabela 02), e o OE do manjericão na mesma concentração, $0,5 \mathrm{mg} / \mathrm{mL}$ (Tabela 04), verificamos que o OE do manjericão $(77,28 \%)$ apresenta uma atividade equiparada ao padrão $(88,55 \%)$ de sequestro de radicais livres. O OE do manjericão mostra-se como um antioxidante tão bom quanto a vitamina $\mathrm{C}$.

Tabela 04: Resultado do teste de atividade antioxidante do Óleo Essencial de Manjericão (Ocimum basilicum L.) com seu percentual de sequestro de radicais livres (inibição)

\begin{tabular}{|c|c|c|c|c|c|c|c|}
\hline \multirow{2}{*}{ Concentração $(\mathrm{mg} / \mathrm{ml})$} & \multirow{2}{*}{$\begin{array}{l}\text { Com. } \\
\text { (ppm) }\end{array}$} & \multirow[t]{2}{*}{ \%Inib. } & \multicolumn{3}{|c|}{ Abs. } & Abs. & \\
\hline & & & $\mathrm{x}$ & $\mathrm{y}$ & $\mathrm{Z}$ & $\mathrm{x}, \mathrm{y}, \mathrm{z}$ & $\mathrm{r}$ \\
\hline 1 & 1000 & 87,728 & 0,025 & 0,024 & 0,025 & 0,025 & 0,000577 \\
\hline
\end{tabular}




\begin{tabular}{|c|c|c|c|c|c|c|c|}
\hline 0,5 & 500 & 77,280 & 0,046 & 0,046 & 0,045 & 0,045 & 0,000577 \\
\hline 0,25 & 250 & 61,194 & 0,078 & 0,079 & 0,077 & 0,077 & 0,001 \\
\hline 0,125 & 125 & 37,811 & 0,124 & 0,126 & 0,125 & 0,125 & 0,001 \\
\hline 0,0625 & 62,5 & 27,032 & 0,148 & 0,146 & 0,146 & 0,146 & 0,001155 \\
\hline DPPH & & & 0,201 & & & & \\
\hline
\end{tabular}

Fonte: Própria (2019)

Atividade Antioxidante: Capim-limão

Para este teste, o método utilizado foi o método do sequestro de radicais livres que foi realizado em quatro concentrações (Tabela 05). O óleo apresentou um bom resultado frente ao sequestro dos radicais livres com um percentual de 47,5\% para a concentração de $1 \mathrm{mg} / \mathrm{mL}$, quando comparamos ao padrão, vitamina $\mathrm{C}$ (Tabela 02). O OE de capimlimão apresenta-se como um antioxidante de atividade intermediária.

Tabela 05: Resultado do teste de atividade antioxidante do OE de capim limão (Cymbopogon citratus) com seu percentual de sequestro de radicais livres (inibição)

\begin{tabular}{|c|c|c|c|c|c|c|c|}
\hline \multirow[t]{2}{*}{ Concentração(mg/ml) } & \multirow{2}{*}{$\begin{array}{l}\text { Com. } \\
\text { (ppm) }\end{array}$} & \multirow[t]{2}{*}{ \%Inib. } & \multicolumn{3}{|c|}{ Abs. } & \multirow{2}{*}{$\begin{array}{l}\text { Abs. } \\
x, y, z\end{array}$} & \multirow[b]{2}{*}{$\mathrm{r}$} \\
\hline & & & $\mathrm{x}$ & $\mathrm{y}$ & $\mathrm{Z}$ & & \\
\hline 1 & 1000 & 47,475 & 0,121 & 0,121 & 0,122 & 0,121 & 0,000577 \\
\hline 0,5 & 500 & 34,055 & 0,153 & 0,153 & 0,152 & 0,152 & 0,000577 \\
\hline 0,25 & 250 & 9,524 & 0,210 & 0,209 & 0,208 & 0,209 & 0,001 \\
\hline 0,125 & 125 & 3,608 & 0,223 & 0,223 & 0,222 & 0,223 & 0,000577 \\
\hline & & & & & & & \\
\hline DPPH & & & 0,231 & & & & \\
\hline
\end{tabular}

Fonte: Própria (2019)

\section{Conclusão}

O estudo do potencial antioxidante de óleos essenciais das espécies Gonçalo Alves (Astronium fraxinifolium), Cidreira (Melissa officinalis), Manjericão (Ocimum basilicum 


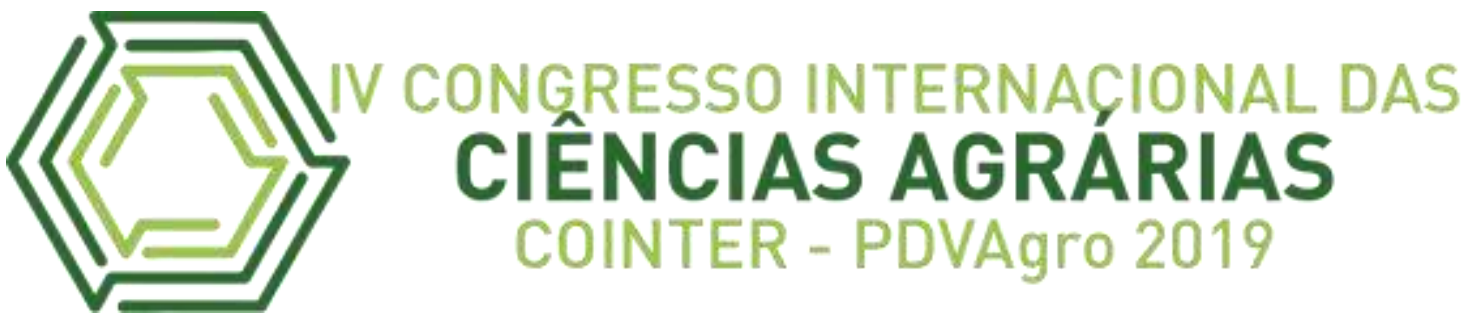

L.) e Capim-limão (Cymbopogon citratus), nos possibilita analisar que o OE de Cidreira apresenta um baixo resultado para o teste de atividade antioxidante frente ao sequestro de radicais livres, com um percentual de 33,5 \% para concentração de $1 \mathrm{mg} / \mathrm{mL}$, quando comparado ao padrão Vitamina $\mathrm{C}$ que apresenta um percentual de $88,5 \%$ para a concentração de $0,5 \mathrm{mg} / \mathrm{mL}$. O OE do Gonçalo Alves apresenta uma péssima atividade antioxidante, seu percentual é de 3,6 \% de sequestro de radicais livres à uma contração de $0,5 \mathrm{mg} / \mathrm{ml}$.

O OE de capim limão apresenta um bom resultado frente ao sequestro dos radicais livres com um percentual de 47,5\% para a concentração de $1 \mathrm{mg} / \mathrm{mL}$, quando comparado ao padrão.

O óleo essencial de manjericão apresenta uma excelente atividade antioxidante frente ao sequestro dos radicais livres com um percentual de $87,7 \%$ para a concentração de $1 \mathrm{mg} / \mathrm{mL}$. Quando comparado ao padrão, vitamina C, e o OE do manjericão, na mesma concentração de $0,5 \mathrm{mg} / \mathrm{mL}$, o OE do manjericão $(77,28 \%)$ apresenta uma atividade equiparada ao padrão $(88,55 \%)$ de sequestro de radicais livres. Portanto, o óleo essencial do manjericão apresenta-se como melhor potencial fitoterápico para o combate de radicais livres, sendo um antioxidante tão bom quanto a vitamina $\mathrm{C}$.

\section{Referências}

BIZZO, H. R.; HOVEL, A. M. C.; RESENDE, C. M. Óleos Essenciais no Brasil: Aspectos Gerais, Desenvolvimento e Perspectivas. Química Nova, v.32, n.3, p.588594, 2009.

BRASILEIRO, B.G; Plantas medicinais utilizadas pela população atendida no "Programa de Saúde da Família", Governador Valadares, MG, Brasil. Rev. Bras. Cienc. Farm. vol.44 no.4 São Paulo Oct./Dec. 2008.

BRITO, A, M G. Aromaterapia: da gênese a atualidade Rev. Bras. Pl. Med., Campinas, v.15, n.4, p.789-793, 2013

BRUNING, M. C. R et al. A utilização da fitoterapia e de plantas medicinais em unidades básicas de saúde nos municípios de Cascavel e Foz do Iguaçu - Paraná: a visão dos profissionais de saúde. Paraná: Ciência \& Saúde Coletiva, 2012.

DAVID, E. F. S. Rendimento e composição do óleo essencial de Mentha piperita L., cultivada em solução nutritiva com diferentes níveis de fósforo. Revista Brasileira de Plantas Medicinais. 2006; 8(4): 183:188. 


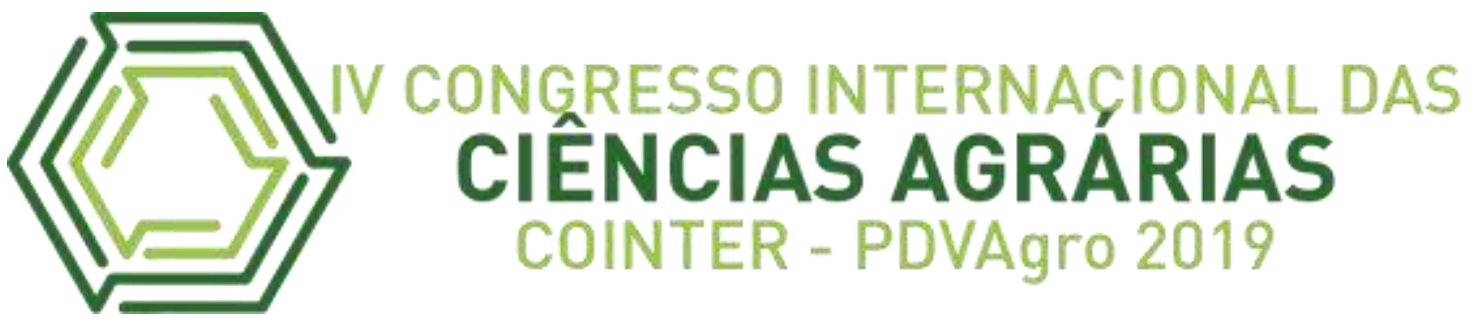

GOBBO-NETO, L.; LOPES, N. P. Plantas medicinais: fatores de influência no conteúdo de metabólitos secundários. Química Nova, v. 30, n. 2, p. 374-381, 2007.

HEGAZI, A. G.; HADY, F. K. A. Egyptian Propolis: 3. Antioxidant, Antimicrobial Activities and Chemical Composition of Propolis from Reclaimed Lands. Zeitschrift für Naturforschung, v.57c, p.395-402, 2002.

KORAĆ R. R. et ak. Potential of herbs in skin protection from ultraviolet radiation. Pharmacognosy Reviews.v.5, n.10, p.164-73, 2011.

MIRANDA, C.A.S. F. Essential oils from leaves of various species: antioxidant and antibacterial properties on growth in pathogenic species. Rev. Ciênc. Agron. vol.47 no.1 Fortaleza Jan./Mar. 2016

RODRIGUES, L.S. NONI (Morinda citrifolia Linn.): determinação fitoquímica e potencial antioxidante pelo método DPPH. Conexões Ciencia e Tecnologia. FortalezaCE, v. 11, n. 4, p. 47-54, dez 2017.

ROESLER, R. et al. Atividade antioxidante de frutas do cerrado. Ciênc. Tecnol. Aliment. vol.27 no.1 Campinas Jan./Mar. 2007.

SOLÓRZANO-SANTOS, F.; MIRANDA-NOVALES, M.G. Essential oils from aromatic herbs as antimicrobial agents. Current Opinion in Biotechnology, v. 23, n. 2, p. $136-141,2012$. 